\title{
Numerical Analysis of Fluid Flow and Heat Transfer for Different Fin Designs and Arrangements of Ceramic Plate- Fin High Temperature Heat Exchanger - Part II
}

\author{
Vijaisri Nagarajan ${ }^{a}$, Yitung Chena*, Quuwang Wang ${ }^{\mathrm{b}}$, Ting Mab \\ a Department of Mechanical Engineering, University of Nevada, Las Vegas, NV 89154-4027, USA \\ ${ }^{\mathrm{b}}$ Key Laboratory of Thermo-Fluid Science and Engineering, MOE, Xi'an Jiaotong University, Xi'an, \\ Shaanxi, 710049, China
}

\begin{abstract}
In this study numerical analysis is carried out for four different types of fins for top and bottom fin arrangement. The obtained results are compared with each other and the design with best heat transfer and minimum pressure drop is selected. The working fluids used in the model are sulfur trioxide, sulfur dioxide, oxygen and water vapor. The operating pressure is $1.5 \mathrm{MPa}$ and the operating temperature ranges from $973 \mathrm{~K}$ to $1223 \mathrm{~K}$. From the results it was found that the ripsaw fin design with thickness of $0.00005 \mathrm{~m}$ gives a good heat transfer rate with minimum pressure drop.
\end{abstract}

The inverted bolt fins also gives a good heat transfer rate but due to the fin arrangement and the flow disturbances caused by the arrangement the pressure drop is the highest compared to other fins. The pressure drop and the heat transfer obtained for the rectangular and triangular fins are similar to each other. Friction factor, Colburn j- factor and dimensionless numbers like Nusselt number, Schmidt number are calculated for all the models. The average Nusselt number obtained for the ripsaw fin design with thickness of $0.00005 \mathrm{~m}$ for the top and bottom arrangement is 3.023 . The friction factor for the ripsaw fins for the top and bottom arrangement is 0.589 .

Keywords - Heat transfer enhancement factor, Ceramic Plate-Fin (PFHE) Heat Exchanger, Nusselt number, Schmidt number, pressure drop.

\section{INTRODUCTION}

Compact heat exchangers (CHE) plays an important role in the field of aerospace, transportation, nuclear and other industries. The need for lightweight, space saving and economical heat exchangers have driven to the development of compact heat exchangers. Surface area density of greater than $700 \mathrm{~m} 2 / \mathrm{m} 3$ is achieved by incorporating fins, ribs tec. The book by Hesselgreaves [1] describes different types of compact heat exchangers like plate-fin heat exchangers, spiral heat exchangers, printed circuit heat exchangers, tube fin heat exchangers etc. A plate-fin heat exchanger is a form of compact heat exchanger made of block of alternating layers of corrugated fins separated by parting sheets. Surface interruption prevents the continuous growth of the thermal boundary layer by periodically interrupting it. Thus the thicker thermal boundary layer which offers high thermal resistance to heat transfer are maintained thin and their resistance to heat transfer is reduced.

In a plate-fin heat exchanger, fins are easily rearranged resulting in cross-flow, counter-flow, cross-counter-flow or parallel flow arrangements. From the research done by Kayansayan [2] the effect of the performance of plate-fin and tube cross-flow heat exchangers due to the outer surface geometry was considered. In this study 10 geometrical configurations were tested and the Reynolds number was varied from 2,000 to 30,000 .

The results showed that the heat transfer coefficient strongly depends on the finning factor $\varepsilon$ and the value of $\varepsilon$ increases with decrease in j-factor. Ranganayakaulu \& Seetharamu [3] carried out an analysis of a cross-flow compact plate-fin heat exchanger for the combined effects of twodimensional longitudinal heat conduction through the exchanger wall, flow non-uniformity and temperature distribution was carried out using the finite element method. The exchanger effectiveness and thermal deterioration due to these effects were studied for various design and operating conditions. Wen \& $\mathrm{Li}[4]$ proposed a study in order to enhance the uniformity of flow distribution. In their study an improved header configuration of plate-fin heat exchanger was proposed. The results showed that the fluid flow maldistribution was very severe in the direction of header length for the conventional header used in the 
industry due to poor header configuration. Manglik \& Bergles [5] studied the heat transfer and pressure drop correlations for the rectangular offset strip-fin compact heat exchanger. The $f$ and $j$ parameters were also found for laminar, transition and turbulent flow regimes. Steady state three-dimensional numerical model was used to study the heat transfer and pressure drop characteristics of an offset strip-fin heat exchanger by Bhowmik \& Lee [6] Three different performance criteria for heat exchangers were tested for different fluids and the appropriate performance criteria for $\operatorname{Pr}=7$ and $\operatorname{Pr}=50$ were found to be $\mathrm{JF}$ (thermal-hydraulic performance factor) and j/f1/3.

Research has been carried out by Ma et al. [7] to find heat transfer and pressure drop performances of ribbed channels in the high temperature heat exchanger. From the results it was found that the Nusselt number and the friction factor were unsuitable to compare heat transfer and pressure drop performances at different temperature conditions. Schulte-Fischedick et al. [8] proposed a ceramic high temperature plate-fin heat exchanger for externally fired combustion process. Thermal performance and pressure drop in ceramic heat exchanger was evaluated using CFD simulations by Monteiro et al. [9].

Correlations for the Colburn and the friction factors for the Reynolds number ranging from 500 to 1500 were evaluated. Simulations with conjugate heat transfer were conducted and the results show the influence of mass flow rate on pressure drop and effectiveness of the heat exchanger. Ponyavin et al. [10] carried out a numerical analysis on the threedimensional computational model of the ceramic high-temperature heat exchanger to investigate fluid flow, heat transfer, and chemical reaction and stress analysis within the decomposer. A decomposition rate of $0.515 \%$ was achieved for $\mathrm{SO} 3$ using this design.

In this study numerical analysis of ceramic plate fin high temperature heat exchanger was carried out to investigate the fluid flow and heat transfer for different fin designs and fin arrangements. This study is an extension of the work done by Nagarajan et al. [11]. In order to save computational space and time a single channel model of the heat exchanger is modeled and studied. The main operating parameters for the heat exchanger design models for the current study are taken from the research done by Ponyavin et al. [10].

\section{GEOMETRY OF THE MODEL AND MATERIAL PROPERTIES}

In this study three-dimensional study of the fluid flow and heat transfer on four type of fins namely rectangular, triangular, inverted bolt fins and ripsaw fins for top and bottom arrangement is carried out. The computer aided geometry (CAD) geometry for all the models are modeled in Solid works [12] and simulations are carried out in ANSYS FLUENT 14.5. The geometry and dimensions for the current study is taken from the work done by Ponyavin et al. [10] and from his study it was found that mass flow rate in all channels can be made almost uniform with a proper design of manifold channels. Hence by applying that concept a single channel model is developed to reduce computational time and memory. Singlebanking configuration is used where in the hot and the cold plates are stacked alternatively.

The current study has hot fluid channel, cold fluid channel and two silicon carbide (SiC) solid regions. Helium fluid flows through the hot channel placed above the solid region and mixture of sulfur trioxide, sulfur dioxide, oxygen and water vapor flows through the cold fluid channel which is placed between the two solid regions. The flow is counter flow where the hot and the cold fluids enter the model from $-x$ and $+x$ directions and exit in the $+x$ and $-x$ directions, respectively. Figure 1 shows the geometry and dimensions of the single channel of plate fin heat exchanger.

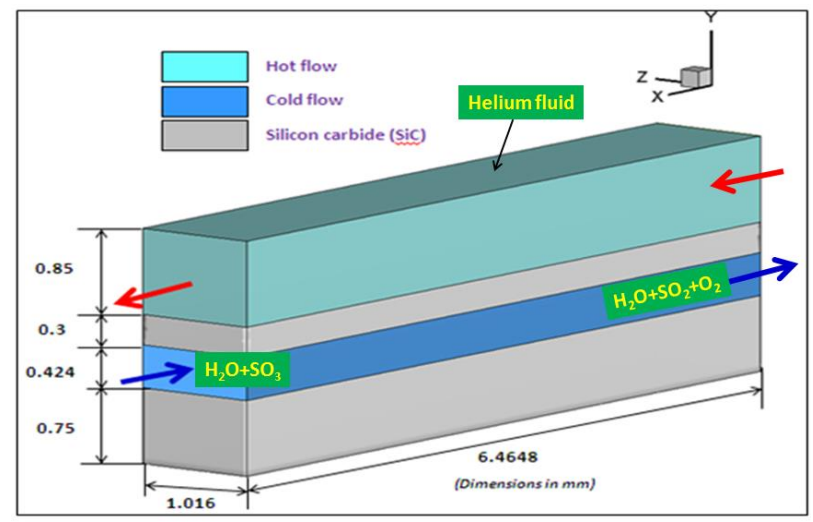

Fig .1. Geometry and dimensions of the channel

To enhance the thermal performance of the PFHE many fin designs are explored and studied. Table 1 shows the dimensions of all the fins 
Table 1. Dimensions of various fins

\begin{tabular}{|c|c|}
\hline Geometry & Definition \\
\hline $\begin{array}{c}\text { Rectangular } \\
\text { fins }\end{array}$ & Height $=0.4 \mathrm{~mm}, \begin{array}{l}\text { length }=0.4 \mathrm{~mm}, \text { width }= \\
0.2 \mathrm{~mm}\end{array}$ \\
\hline $\begin{array}{c}\text { Triangular } \\
\text { fins }\end{array}$ & $\begin{array}{c}\text { Height }=0.3 \mathrm{~mm}, \text { breadth }=0.2 \mathrm{~mm}, \text { length }= \\
0.4 \mathrm{~mm}\end{array}$ \\
\hline $\begin{array}{c}\text { Inverted bolt } \\
\text { type fins }\end{array}$ & $\begin{array}{c}\text { Diameter of top cylinder }=0.2 \mathrm{~mm}, \text { diameter } \\
\text { of bottom cylinder }=0.4 \mathrm{~mm}\end{array}$ \\
\hline $\begin{array}{c}\text { Ripsaw fins } \\
\text { Larger height of the rectangle }=0.3 \mathrm{~mm}, \\
\text { smaller height of the rectangle }=0.15 \mathrm{~mm}, \\
\text { thickness }=0.05 \mathrm{~m}\end{array}$ \\
\hline
\end{tabular}

In order to avoid backflow and provide entrance zone for each channel no fins are placed near the inlet and the outlet region on the cold channel. A study was carried out by increasing the length of the inlet and outlet to 5,10 and 15 times the hydraulic diameter. At 10 times the hydraulic diameter the flow becomes fully developed and hence the length of the inlet and outlet are taken to be 10 times the hydraulic diameter. The material properties, boundary and operating conditions are taken from the work done by Nagarajan et al. [11].

\section{NUMERICAL METHOD AND ALGORITHM}

The finite volume method is one of the most versatile discretization techniques used in CFD. The governing equations are solved in the Cartesian coordinate system using a control volume finite difference method that is similar to the approach introduced by Patankar [14]. ANSYS FLUENT [13] a commercial CFD program based on the finite volume method is among the most powerful packages of existing software used for solving fluid flow and heat transfer problems. The pressure-based segregated solution algorithm is used for the given problem. It can be simply described as the process of solving the governing equations in a sequential order as opposed to simultaneously as with a coupled solver.

The geometry of the model is meshed in ANSYS WORKBENCH [13] mesh generator. Hexahedral elements are used for meshing. The mesh is refined near the walls particularly for the cold flow channel with fins. The mesh refinement near the wall helps in calculating the fluid flow and heat transfer properties accurately. In order to check the mesh dependence on fluid flow and heat transfer properties, the grid independent study was done for both staggered and top and bottom arrangement. From the study, optimum nodes with difference in pressure drop and heat transfer of less than $5 \%$ is selected for further study. Around 481,558 cells, 1,496,152 faces and 532,599 nodes are selected for further study for all the cases.The friction factor, the Colburn factor and the Prandtl number are calculated using the formula shown below:

$$
\begin{aligned}
& f=\frac{\left(\frac{\Delta P}{L}\right) * D_{h}}{\left(0.5 \cdot \rho \cdot U^{2}\right)} \\
& j=\frac{\overline{N u}}{\operatorname{Re} \cdot \operatorname{Pr}^{\frac{1}{3}}} \\
& \operatorname{Pr}=\frac{\mu \cdot C_{p}}{K}
\end{aligned}
$$

The Schmidt number which is the ratio of the viscous diffusion rate to the mass diffusion rate is calculated based on the average temperature of the reacting channel. The validation of the fluid flow and heat transfer for the rectangular and ripsaw fins was carried out in the previous study done by Nagarajan et al. [11]. The friction factor was compared with the published result by Manson [15] and the Colburn factor was compared with the correlation published by Wieting [16]. The obtained CFD result was in good agreement with the published result and the slight offset is due to the difference in the dimensions of the geometry. Since the numerical results agree closely with the published results and follow the same trend, further research is carried out for the selected design.

\section{RESULTS AND DISCUSSIONS}

\section{A. Fins Arranged on the Top and the Bottom Solid}

In this study, the fins are arranged on both the top and the bottom solid regions to enhance the heat transfer. It is found that since the bottom solid region doesn't have any heat input and the heat transfer is only from top solid region there is a little increase in heat transfer rate compared to the uniform arrangement. The pressure drop obtained is higher compared to the other two arrangements because there is an increase in heat transfer surface area and more flow disturbances produced by the fins. 


\section{Case 1 (Single Channel Model with Rectangular} Fins)

In this study the rectangular fins are arranged on both the top and bottom solid regions. The heat transfer surface area of the rectangular fin arrangement is 2.47-10-5 m2. The pressure drop obtained for this model is $56.49 \mathrm{~Pa}$ which is twice higher than the staggered arrangement. The friction and Colburn $\mathrm{j}$ factor is 1.778 and 0.016 , respectively. Due to the top and bottom arrangement there is strong flow disturbance which results in the increase of pressure drop.

Figure 2 shows the pressure and velocity contour for the single channel model with rectangular fins arranged on the top and the bottom solid regions.

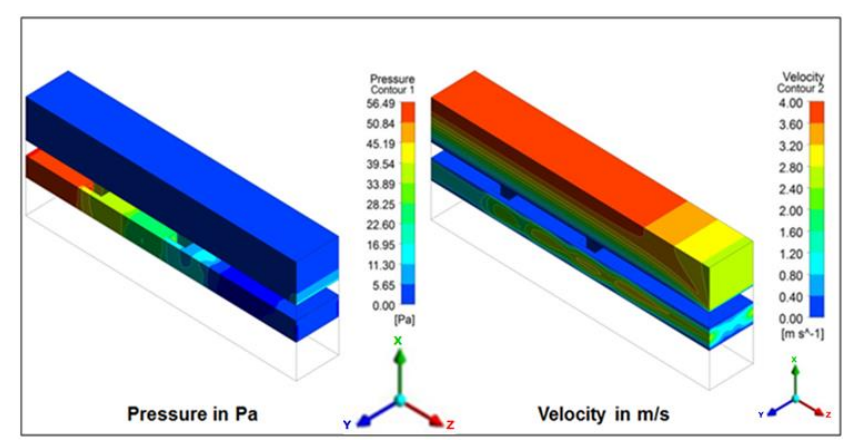

Fig .2. Pressure in $\mathrm{Pa}$ and velocity in $\mathrm{m} / \mathrm{s}$ contour for single channel model with rectangular fins arranged on the top and bottom solid regions

The horseshoe vortex is formed at the beginning of the fins and it extends along the sides. Recirculation is formed behind the fins in the wake region. The shear layer separates and form twin vortices where one vortex rotates in the clockwise direction and the other in the opposite direction, In Figure 3, the recirculation can be seen clearly in the second and the fourth row of fins but not in the first and the third row. The y-plane is taken at a distance from threefourth of the top fin and from one-fourth of the bottom fin. Hence more recirculation is found on the top fins.

From the streamline along the z-plane recirculation can be found alternately along the top and the bottom fins. Since the Reynolds number is only 244 there is no secondary vortex formed. The recirculation region is located at $0.00108 \mathrm{~m}$ and the reattachment region of the shear layer is located at $0.00201 \mathrm{~m}$.

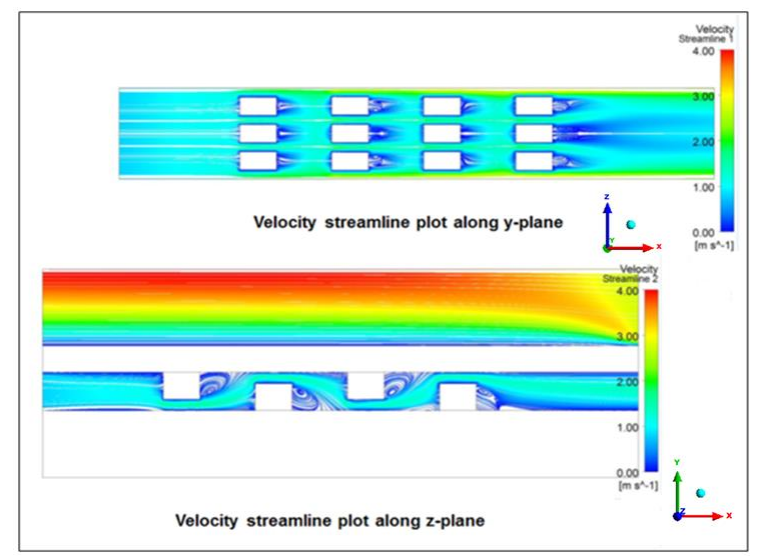

Fig .3. Velocity streamline for the single channel model with rectangular fins arranged on the top and bottom solid regions at $y=0.0032 \mathrm{~m}$ and $\mathrm{z}=0.0053 \mathrm{~m}$

Figure 4 shows the top and bottom arrangement of rectangular fins for pressure and velocity plots. The pressure decreases uniformly from inlet to P1 where the fins start. Pressure is high at P1 and it starts decreasing and reaches minimum at P2. Similarly the velocity increases as the fluid flow around the fins and becomes maximum at P2. Recirculation zone is formed behind the top fin and adverse pressure gradient is formed. Hence pressure increases at P3 and the velocity is minimum at P3.

The bottom fin starts and the pressure is low at P4 where there is no obstacle and the velocity increases. The pressure decreases as the fluid travels to the rear of the fins. The process continues till the end of the fourth fin and the pressure and velocity becomes stable till it reaches the outlet.

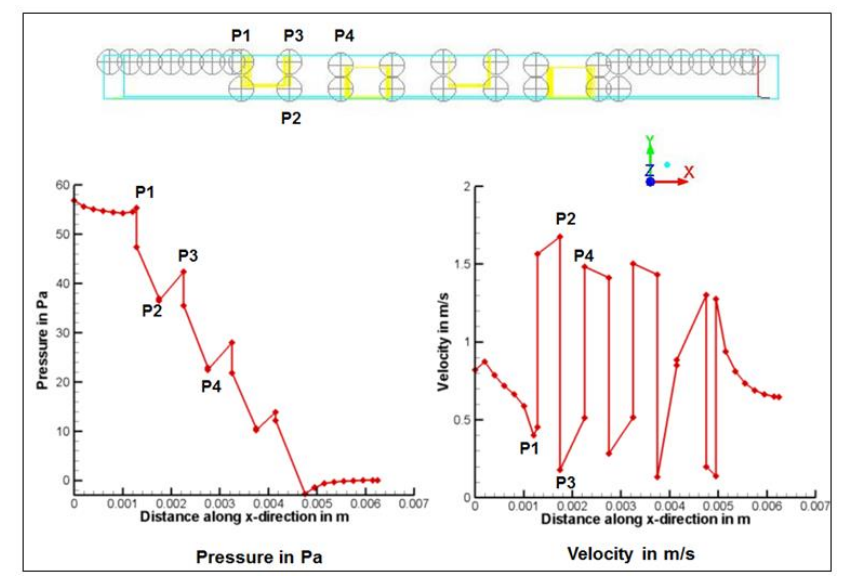

Fig .4. Pressure and velocity for single channel model with rectangular fins arranged on the top and bottom solid regions 
Figure 5 shows the temperature and the local heat transfer coefficient for the rectangular fins. The temperature and the local heat transfer coefficient increases until it reaches P1. The local heat transfer coefficient decreases at P2, P3 which are placed away from the hot solid wall.

The temperature becomes high at P4. The fins placed on the bottom solid are away from the heat source and hence the temperature and the local heat transfer coefficient at P5 and P6 are small. The heat flux is high at the upper solid wall and it decreases away from the hot wall. The temperature increases at P7 where the top fin start and again decreases at P8. Away from the wall the temperature difference increases resulting in the decrease of the heat transfer coefficient.

The process repeats till the end of the fins and the temperature and the local heat transfer coefficient increases till the fluid reaches the outlet. Due to the increase in the heat transfer surface area, the obtained heat transfer rate is high compared to the uniform arrangement of rectangular fins.

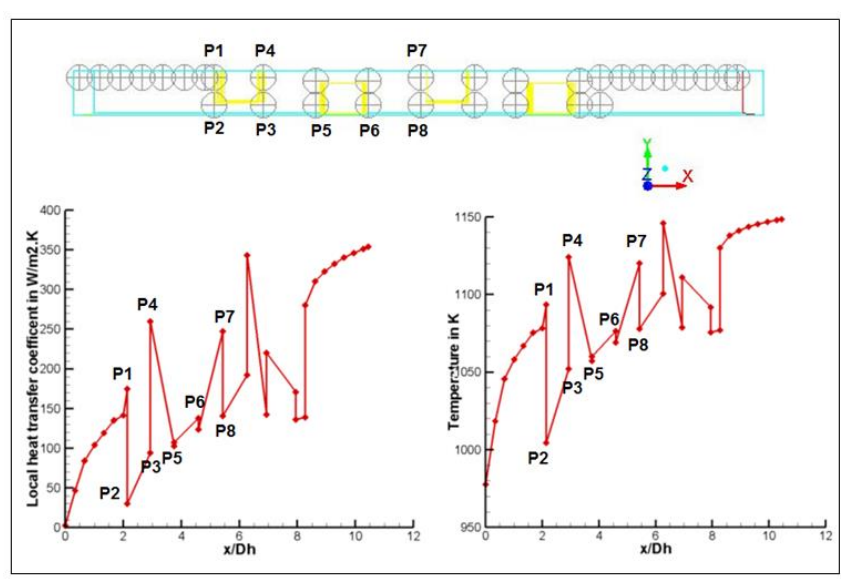

Fig .5. Temperature and local heat transfer coefficient of single channel model with rectangular fins arranged in the top and bottom solid regions

2. Case 2 (Single Channel Model with Triangular Fins)

Two rows with three triangular fins arranged in alternate manner along the top and bottom solid regions are studied. The heat transfer surface area of triangular fins is smaller than rectangular fins. The heat transfer surface area for the top and bottom fin arrangement is $2.342 \cdot 10-5 \mathrm{~m} 2$. The pressure drop and the friction factor for this model are $34.04 \mathrm{~Pa}$ and
1.073, respectively. The average Nusselt number and the Colburn j-factor is 3.099 and 0.016 , respectively. The obtained heat transfer rate and the pressure drop is less than the rectangular fins.

Figure 6 shows the velocity streamline for the triangular fins along $y$ and $z$-planes. The obtained streamlines are similar to the rectangular fins. Recirculation zone is found in the wake region forming symmetrical twin vortices behind each fin. The recirculation region is located at $0.00123 \mathrm{~m}$ and the reattachment region is located at $0.00207 \mathrm{~m}$.

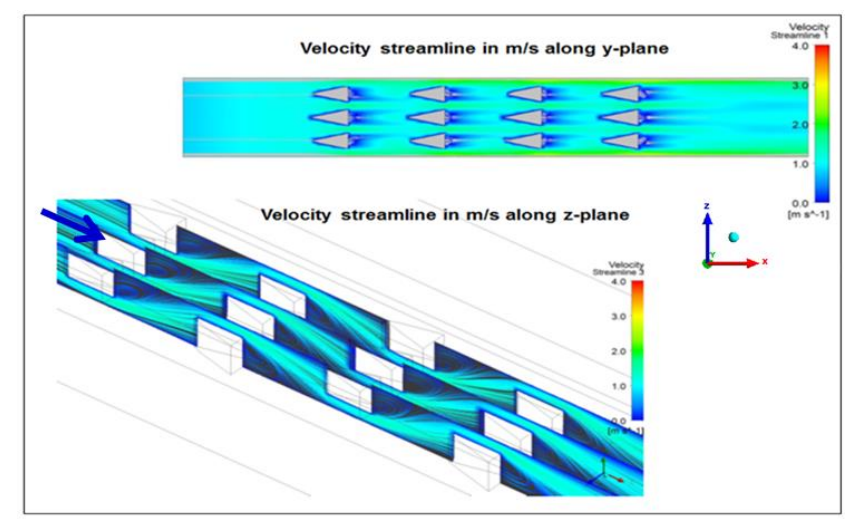

Fig .6. Velocity streamline for single channel model with triangular fins arranged on the top and bottom solid regions at $y=0.0045 \mathrm{~m}$ and $\mathrm{z}=0.0059 \mathrm{~m}$

It can be seen that recirculation zone is formed behind the top and bottom fins alternately. Since the Reynolds number is low there is no formation of secondary vortex. Though the bottom solid does not have any heat input the heat transfer rate of this model is more than the staggered triangular fin arrangement. The increase in heat transfer is due to the increase in heat transfer surface area. The pressure, velocity and local heat transfer coefficient plot for the triangular fins are similar to the rectangular fin model and hence not shown in this study.

3. Case 3 (Single Channel Model with Inverted Bolt Type Fins)

The obtained heat transfer rate is higher than the triangular and rectangular fins. The heat transfer surface area is $2.438 \cdot 10-5 \mathrm{~m} 2$ which is higher than all the fins. The only disadvantage of this fin type is the high pressure drop and the friction factor. Due to the complex design and arrangement horseshoe vortices are formed which increases the pressure drop and the friction factor. 
The obtained local heat transfer coefficient and the average Nusselt number are $221 \mathrm{~W} / \mathrm{m} 2 \cdot \mathrm{K}$ and 3.16 , respectively. The pressure and velocity for the inverted bolt fin are similar to the rectangular and triangular fins. The velocity streamline plot for the inverted fin mounted on the top and the bottom solids is shown in Figure 7. A strong horseshoe vortex is formed in front of the cylindrical fins and fluid flows around the cylinder to the rear side. The shear layers starts to separate and recirculation zone is formed in the rear side of the top fins for the top fins and in the rear side of the bottom fins for the bottom fins.

The recirculation zone covers almost three-fourth of the space between the fins and the shear layers reattach to the wall after the wake region. Again flow separation occurs and this results in periodic breaking of the boundary layer thus enhancing the heat transfer. The recirculation region is located at $0.00123 \mathrm{~m}$ and the reattachment region of the shear layer is located at $0.001978 \mathrm{~m}$.

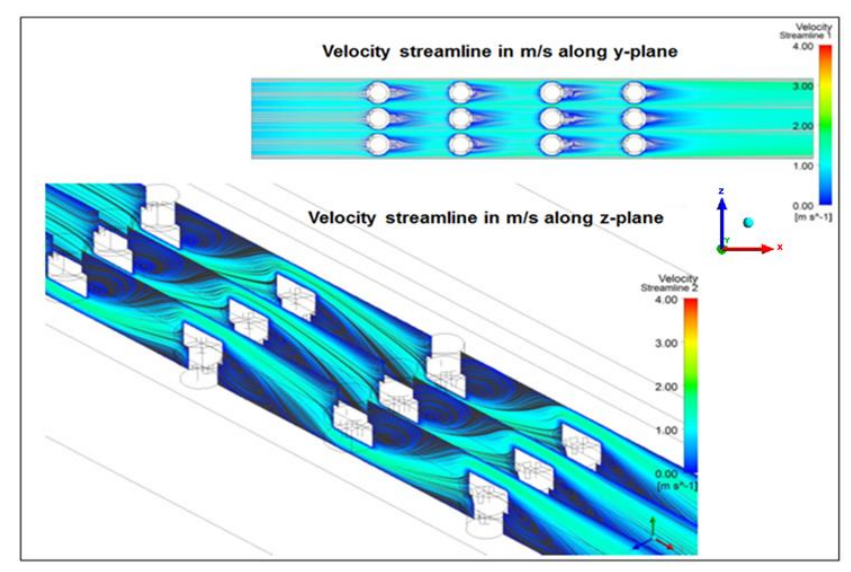

Fig .7. Velocity streamline for the single channel model with inverted bolt fins arranged on the top and bottom solid regions at $\mathrm{y}=0.0036 \mathrm{~m}$ and $\mathrm{z}=0.0045 \mathrm{~m}$

Figure 8 shows the velocity and local heat transfer coefficient. The velocity along the flow is uniform till it reaches the fins. The velocity is low at $\mathrm{P} 1$ and the pressure is high. The velocity increases at P2 as the fluid flow around the fins and it reaches a maximum at P3. The pressure is least at this point. Recirculation takes place at the rear of the fins and the velocity is minimum at P4 and the pressure is maximum at P4. The fluid then flows through the bottom fins and the velocity is minimum at P5 and pressure is high at this point. The fluid velocity then reaches maximum at $P 6$ and the pressure is minimum at $\mathrm{P} 6$.
The process continues until it reaches the fourth row of the fins and it becomes stable after the end of the fins. The local heat transfer coefficient is high for the top fins which are in contact with the solid wall. The temperature and local heat transfer coefficient is low at P2, P3, P5, etc. which are away from the hot solid surface. After the fourth tow of the fin the temperature increases without much fluctuation till reaches the outlet.

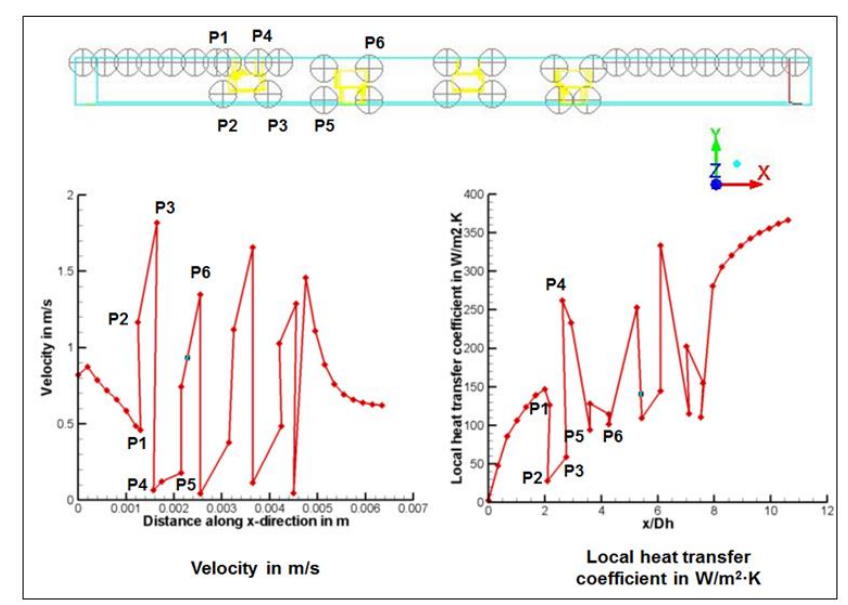

Fig .8. Velocity and local heat transfer coefficient for the single channel model with inverted bolt fins arranged on the top and bottom solid regions

4. Case 4 (Single Channel Model with Ripsaw Fin Thickness of $0.00005 \mathrm{~m}$ )

The last model studied in this research is the ripsaw fin thickness of $0.00005 \mathrm{~m}$ arranged on the top and bottom solid regions. The heat transfer surface area is $2.371 \cdot 10-5 \mathrm{~m} 2$. This type of arrangement has no recirculation due to their shape. As a result of this the obtained pressure drop and the friction factor is also less. The obtained friction factor and the pressure drop is 0.589 and $18.73 \mathrm{~Pa}$, respectively.

The obtained average heat transfer coefficient and the average Nusselt number is $211.43 \mathrm{~W} / \mathrm{m} 2 \cdot \mathrm{K}$ and 3.023 , respectively. The streamline velocity plot shows that there is no recirculation the streamlines are parallel to the flow direction. The obtained velocity and local heat transfer coefficient is similar to the other fins explained above. Figure 9 shows the velocity streamline along the $y$ and $z$-planes. 


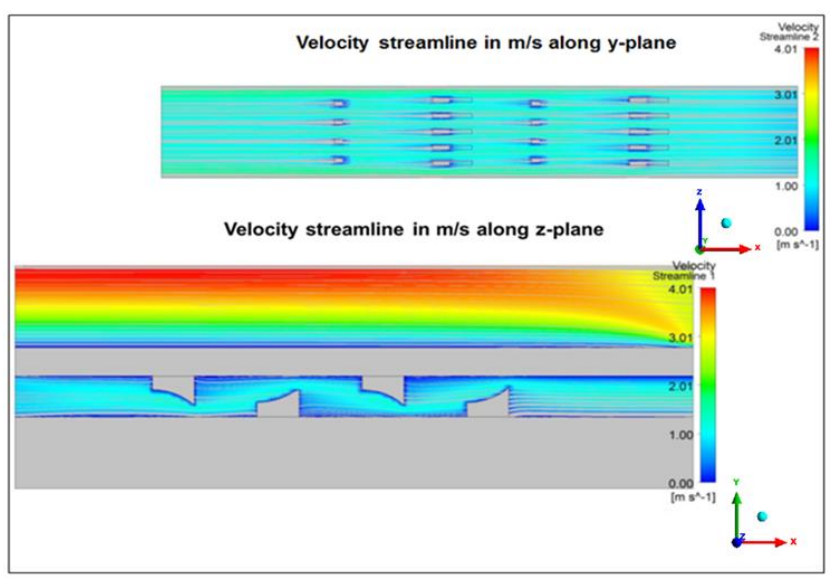

Fig .9. Velocity streamline for single channel model with ripsaw fin of thickness $0.00005 \mathrm{~m}$ arranged on the top and bottom solid regions at $\mathrm{y}=0.0032 \mathrm{~m}$ and $\mathrm{y}=0.0046 \mathrm{~m}$

The pressure, temperature and local heat transfer coefficient plots are similar to the other fin types. The velocity is low and the pressure is high at the stagnation point and in the recirculation region. The temperature and the local heat transfer coefficient are high at places near the top solid wall and low at regions away from the hot solid wall.

From the results it is found that there is not significant increase in heat transfer for the top and bottom fin arrangement. The reason is because the bottom solid has no heat input and the heat is transferred only from the top solid. In the top and bottom arrangement there are only 6 fins placed in the hot solid wall and 6 placed in the cold solid wall. Hence the obtained heat transfer is less than the regular staggered arrangement of the fins.

In real case, the channel arrangement is periodic and the hot helium fluid flows below the cold solid wall and heat transfer will be increased by having fins on both the solid walls. However in the current study the hot flow channel is present only above the reacting channel and hence the heat transfer obtained is less. The obtained average Nusselt number and the Colburn $j$ factor is similar to the uniform fin arrangement. The friction factor is increased and the ripsaw fin with thickness of $0.00005 \mathrm{~m}$ is found to have the lowest friction factor.

The effectiveness of the heat exchanger increases for the top and bottom arrangement. The pressure drop increases due to the presence of fins on both the top and bottom solids. In this arrangement recirculation and vortices are formed which increases the pressure drop and friction factor. The pressure drop is the highest for rectangular fins and least for the ripsaw fins. Hence ripsaw fin with thickness of $0.00005 \mathrm{~m}$ is considered to be the best design because it gives good heat transfer with minimum pressure drop. The obtained effectiveness is around 52 to $54 \%$ for all the fin designs. The heat transfer surface area is less and hence the effectiveness of the heat exchanger is less. The length of the heat exchanger channel with staggered rectangular, triangular and ripsaw fin with thickness of $0.00005 \mathrm{~m}$ is increased from 0.0064648 $\mathrm{m}$ to $0.064648 \mathrm{~m}$.

The heat transfer area of the long staggered rectangular fins is $2.44 \cdot 10-4 \mathrm{~m} 2$, triangular fins is 2.35.10-4 $\mathrm{m} 2$ and the ripsaws with thickness of $0.00005 \mathrm{~m}$ is $2.4354 \cdot 10-4 \mathrm{~m} 2$. The obtained heat exchanger effectiveness for rectangular fin is $80.15 \%$, triangular fin is $87.25 \%$ and ripsaw fins with thickness of $0.00005 \mathrm{~m}$ is $92.13 \%$.

\section{v. CONCLUSION}

The obtained average Nusselt number is higher for inverted bolt fins which is 3.201 followed by triangular fins which is 3.099. The obtained pressure drop for the rectangular fins is higher compared to the triangular fins for similar heat transfer rate. For similar heat transfer rate the obtained pressure drop which is 18.73 $\mathrm{Pa}$ is less for the ripsaw fins compared to the other fins. Hence ripsaw fin with thickness of 0.00005 $\mathrm{m}$ is considered to be the best design with less pressure drop and reasonable heat transfer rate.

The inverted bolt fins have the highest average Nusselt number followed by the triangular fins. But the pressure drop for the rectangular fin which is $56.49 \mathrm{~Pa}$ is almost twice that of the triangular fin which is $34.08 \mathrm{~Pa}$. Eventhough the obtained average Nusselt number for the ripsaw fin with $0.00005 \mathrm{~m}$ thickness which is $(\mathrm{Nu})^{-}=3.026$ is less compared to the other fins, the obtained pressure drop for the ripsaw fins is the least of all the four fins which is 18.73 $\mathrm{Pa}$.

Hence the ripsaw fin thickness of $0.00005 \mathrm{~m}$ is selected as the best design with good heat transfer rate of $0.475 \mathrm{~W}$ and minimum pressure drop of 18.73 $\mathrm{Pa}$. The effectiveness increased from $52 \%$ for $0.0064648 \mathrm{~m}$ to $82 \%$ for $0.064648 \mathrm{~m}$. 


\section{REFERENCES}

[1] Hesselgreaves, J. E. (2001). Compact Heat Exchangers Selection, Design and Operation. Oxford, UK: Elsevier Science Ltd.

[2] Kayansayan, N. (1994). Heat Transfer Characterization in Plate-Fin Tube Heat Exchangers. International Journal of Refrigeration, 17, pp. 49-57.

[3] Ranganayakulu, C. \& Seetharamu. K.N. (1999). The Combined Effects of Wall Longitudinal Heat Conduction, Inlet Fluid Flow Non-Uniformity and Temperature Non-Uniformity in Compact TubeFin Heat Exchangers: A Finite Element Method. International Journal of Heat and Mass Transfer, 42, pp. 263-73.

[4] Wen, J. \& Liu. (2004). Study of Flow Distribution and Its Improvement on The Header of Plate-Fin Heat Exchanger. Cryogenics, 44(11), pp. 823831.

[5] Manglik, R. M. \&. Bergles, A. E. (1995). Heat Transfer and Pressure Drop Correlations for The Rectangular Offset Strip Fin Compact Heat Exchanger. Experimental Thermal and Fluid Science, 10, pp. 171-80.

[6] Bhowmik. H., \&. Lee.K. (2008). Analysis of Heat Transfer and Pressure Drop Characteristics in an Offset Strip Fin Heat Exchanger. International Communications in Heat and Mass Transfer, 36(3), pp. 259-263.

[7] Ma, T., Wang, Q. W., Zeng, M., Chen, Y., Liu, Y., \& Nagarajan, V. (2012). Study on Heat Transfer and Pressure Drop Performances of Ribbed Channel in the High Temperature Heat Exchanger. Applied energy, 99, pp. 393-401.
[8] Schulte-Fischedick, J., Dreibigacker, V., \& Tamme, R. (2007). An Innovative Ceramic High Temperature Plate-Fin Heat Exchanger for EFCC Processes. Applied Thermal Engineering, 27(8-9), pp. 1285-94.

[9] Monteiro, D. \&. B., \& Batista, De Mello, P.E. (2012). Thermal Performance and Pressure Drop in a Ceramic Heat Exchanger Evaluated Using CFD Simulations. Energy, 45, pp. 489496.

[10] Ponyavin, V., Chen, Y., Mohamed, T., Trabia, M.B., Hechanova, A.E., \& Wilson, M. (2012). Design of a Compact Ceramic HighTemperature Heat Exchanger And Chemical Decomposer for Hydrogen Production. Heat Transfer Engineering, 33(10), pp. 853-70.

[11] Nagarajan, V., Chen, Y., Wang, Q., \& Ma, T. (2014). Hydraulic and Thermal Performances of a Novel Configuration Of High Temperature Ceramic Plate-Fin Heat Exchanger. Applied Energy, 113, pp. 589-602.

[12] SOLIDWORKS 2013., SOLIDWORKS Corp.

[13] [ANSYS Inc. (2011). ANSYS 14.5 user's guide.

[14] Patankar, S. (1980). Numerical Heat Transfer and Fluid Flow. New York.

[15] Manson, S. V. (1950). Correlations of Heat Transfer Data and Friction Data for Interrupted Plate Fins Staggered in Successive Rows. Washington DC: National Advisory Committee for Aeronautics.

[16] Wieting, A. R. (1975). Empirical Correlations for Heat Transfer and Flow Friction Characteristics of Rectangular Offset-Fin-Plate-Fin Heat Exchanger. ASME, Int J. Heat Transfer, 97, pp. 480-490. 